\title{
Cross-modal decoupling in temporal attention
}

\section{Stefanie Mühlberg ${ }^{1}$, Giovanni Oriolo $^{1}$, Salvador Soto-Faraco ${ }^{1,2}$}

1 Departament de Tecnologies de la Informació i les Comunicacions, Universitat Pompeu Fabra, Barcelona 08018, Spain

2 ICREA, Institució Catalana de Recerca i Estudis Avançats, Barcelona 08010, Spain

Keywords: behaviour, cross-modal, temporal attention, vision, touch

Corresponding Author: Salvador Soto- Faraco

Affiliation: Departament de Tecnologies de la Informació i les Comunicacions, Universitat Pompeu Fabra, Spain, and Institució Catalana de Recerca i Estudis Avançats (ICREA)

Email: $\quad$ salvador.soto@icrea.cat

Postal address: $\quad$ Departament de Tecnologies de la Informació i les Comunicacions Universitat Pompeu Fabra

Room 55.108

C $\backslash$ Roc Boronat, 138

08018 Barcelona

Spain

Fax number: $\quad$ +3493542 $\quad$ +3517

Words

Total 8360

Abstract 209

Introduction 418 (without citations) 


\begin{abstract}
Prior studies have repeatedly reported behavioural benefits to events occurring at attended, compared to unattended, points in time. It has been suggested that, like for spatial orienting, temporal orienting of attention spreads across sensory modalities in a synergistic fashion. However, the consequences of cross-modal temporal orienting of attention remain poorly understood. One challenge is that the passage of time leads to an increase in event predictability throughout a trial, thus making it difficult to interpret possible effects (or lack thereof). Here we used a design that avoids complete temporal predictability to investigate whether attending to a sensory modality (vision or touch) at a point in time confers beneficial access to events in other, non-attended, sensory modality (touch or vision, respectively). In contrast to previous studies, and different to what happens with spatial attention, we found that events in one (unattended) modality do not automatically benefit from happening at the time point when another modality is expected. Instead, it seems that attention can be deployed in time with relative independence for different sensory modalities. Based on these findings, we argue that temporal orienting of attention can be cross-modally decoupled in order to flexibly react according to the environmental demands, and that the efficiency of this selective decoupling unfolds in time.
\end{abstract}




\section{Introduction}

Attention is paramount to boost the processing of relevant environmental events (Treue, 2003).

For instance, spatial attention grants quicker reactions (Posner et al., 1980), lower perception thresholds (Yeshurun and Carrasco, 1998) and increased accuracy (Luck et al., 1994) for visual events appearing at an attended location. Considering endogenous voluntary attention (Jonides, 1981; Corbetta \& Shulmann, 2002; Carrasco, 2012), these benefits have also been demonstrated with time, rather than space, as selection criterion (e.g., Westheimer \& Ley, 1996; Coull \& Nobre, 1998; Miniussi et al., 1999; Griffin et al., 2001; Correa et al., 2004; Jones, 2004; Nobre et al., 2007; Rohenkohl et al., 2012). In addition, it is widely accepted that orienting spatial attention within one modality benefits processing in other modalities at that location, a pattern known as cross-modal synergy (e.g. Spence \& Driver, 1996; Spence \& Driver, 1997; Driver \& Spence, 1998a,; Spence et al., 1998; Eimer, 1999; Spence et al., 2000; McDonald et al., 2000; Macaluso et al., 2000; Kennett et al., 2001; Spence et al., 2001; Eimer et al., 2002; Macaluso et al., 2002; Krumbholz et al., 2009; Santangelo et al., 2009).

Cross-modal synergies not only exist in spatial attention, but have also been claimed in the domain of temporal attention (e.g. Miniussi et al., 1999; Meredith, 2002). That is, expecting an event in one modality at a certain time might lead to benefits in processing of stimuli presented in a different (unexpected) modality at that instant. Lange \& Röder (2006) addressed this question in an ERP study including behavioural measures where expectancy of an audition or visual event was manipulated across time points (early or late). The authors found that reaction times (RT) were faster not only for stimuli in the expected (primary) modality and temporal interval, but also for stimuli in the unexpected (secondary) modality occurring at the expected time point. Their ERP data showed a modulation of the N100 component for events in 
both, the primary and secondary modality, suggesting a cross-modal synergy in temporal attention. However, like in many other temporal attention studies, events occurring at the later time point were completely predictable (Sanders, 1975) therefore violating the assumption of uncertainty (Coull \& Nobre, 1998; Griffin et al., 2001). This is why Lange and Röder's conclusions are based only on expectancy manipulating at the earlier time point, preventing to investigate the temporal course of attentional modulations.

The present study manipulated relative stimulus probabilities in vision and touch independently, and maintained uncertainty throughout the trial by adding foils. This allowed us to gauge attention effects at early and late time intervals for each modality. If the hypothesis of cross-modal synergy in temporal orienting of attention holds, then one would expect faster RTs for all the stimuli presented at the overall expected time, regardless of modality prevalence (that is, for events in the primary or secondary, less likely, modality). Instead, if such synergistic effects fail to operate, then only the primary modality will be facilitated at the overall expected time points, without coupling of performance in the secondary modality. In this case, one would expect an interaction between modality prevalence and temporal expectation. Moreover, performance in the secondary modality might abide to its relative temporal distribution independently of the primary modality.

\section{Methods}

\section{Participants}

A total of 29 participants volunteered for this experiment ( 2 left-handed; 18 female; mean age 26.62 years, age-range 18-36 yeas) in exchange for $8 €$ per hour. They all reported normal or 
corrected to normal vision and gave written informed consent to participate in the study, which is in accordance to the Declaration of Helsinki and approved by the ethics committee CEIC Parc de Mar (University Pompeu Fabra, Barcelona, Spain).

\section{Stimulus design}

The stimuli could be visual or tactile, and presented as single or double pulse stimulation. Visual stimuli consisted of the illumination of a yellow LED $(0.025 \mathrm{~cd} / \mathrm{m} 2)$ at the centre of a black cardboard box $(32.5 \times 20 \times 11 \mathrm{~cm})$ placed $35 \mathrm{~cm}$ lower frontal viewing distance from the participant (see Figure 1). The single pulse visual stimulus was a flash of 200ms, and the double pulse stimulus consisted of two $75 \mathrm{~ms}$ flashes, separated by a $50 \mathrm{~ms}$ gap. Tactile stimulation was presented on the index finger pad of the participant's hand, which was placed spatially aligned underneath the LED delivering the visual stimuli (left or right hand stimulation was fixed within participant and counterbalanced between them). The tactile stimuli were delivered by a solenoid tapper (round tip, 8 mm, Miniature Solenoid Tapper Controller Mk3, MSTC3-10M, M\&E Solve). For single pulse stimulation, the tapper was lifted for $10 \mathrm{~ms}$; double pulse stimuli consisted of two $10 \mathrm{~ms}$ stimulations, separated by a $30 \mathrm{~ms}$ gap. The tactile stimulation did not cause any pain or annoyance to the participant. These parameters of the visual and tactile stimuli were adjusted based on prior informal pilot testing, so that the behavioural performance for both, reaction times and accuracy, in this task would be roughly equivalent for both modalities.

\section{Experimental design and procedure}

The experiment was conducted and responses were collected using MatLab 7.10.0 (The MathWorks Inc.; Natick, MA, USA). The goal of the study was to study the effects of attention 
to time and modality. To achieve this goal, we adopted a discrimination task, in which we manipulated the participants' expectations about the onset time and modality of an upcoming target. The experiment was conducted in a sound attenuated room with dim illumination $\left(1.595 \mathrm{~cd} / \mathrm{m}^{2}\right)$. Participants sat in an armchair with their hands resting on a table and covered from view by a cardboard box that bore the fixation and stimulation LEDs (Fig. 1A). Every trial started with the delivery of an auditory warning tone $(1000 \mathrm{~Hz}, 100 \mathrm{~ms}, 60 \mathrm{~dB}[\mathrm{~A}])$, via headphones, and was followed by masking white noise $(55 \mathrm{~dB}[\mathrm{~A}])$ throughout the trial. Either $1 \mathrm{~s}$ or $2.5 \mathrm{~s}$ after the warning tone, a target stimulus could appear (Fig. 1B), which could either be visual or tactile and could also be single or double pulse stimulation. The task was to discriminate between single and double pulse stimulation regardless of modality or time point of presentation. Responses were delivered by releasing one of the two foot pedals (toe or heel) to indicate double or single stimulus (respectively). Participants were informed before every block about the most likely time point of target appearance and the most likely modality, but they were also told to always deliver a response and instructed to answer as fast and as accurately as possible. After the response (or after the response timeout of $1.5 \mathrm{~s}$ ), an intertrial interval of $2 \mathrm{~s}$ led to the beginning of the next trial. When no stimulus was presented at one of the possible onset times, then a gap in the background white noise occurred (20ms, 10ms ramps envelope) as provision of additional temporal information.

Within the experiment, the participants' expectations about the onset time and target modality were manipulated by adjusting probabilities for the two factors (Fig. 1C). Temporal expectation was manipulated across blocks of trials, whereas modality probability was a between participants factor. At the beginning of each experimental block, participants were informed which time interval ( $1 \mathrm{~s}$ or $2.5 \mathrm{~s})$ would be more likely to contain the target and we will refer to this point as 
the expected time point. If the early stimulus onset was expected, 55\% of all trials contained a target after $1 \mathrm{~s}$ and $22.5 \%$ of all trials contained a target after $2.5 \mathrm{~s}$. This pattern was inverted for the blocks in which the late stimulus onset was expected. In all cases, $22.5 \%$ of trials in the block were catch trials without a target in either of the time intervals, in which case participants were instructed to withhold the response.

In addition to the temporal attention manipulation described above, attention to modality was manipulated by making one modality were more likely overall (primary; 66\%) than targets in the other modality (secondary; 33\%). Attention towards modality was thereby handled as a betweensubject factor, with 15 participants having vision as their primary modality and 14 participants touch.

Importantly, in order to investigate the distribution of cross-modal attention, trials of the primary and secondary modality were not equally likely throughout time. The primary modality followed the manipulation of temporal attention, through which targets in the primary modality were more likely at the expected time point, $86.4 \%$, vs. $13.6 \%$ at the unexpected time point. For the secondary modality, overall probabilities reversed so that, of all secondary modality targets, only $33.3 \%$ occurred at the expected, and overall more likely, time point of the primary modality and $66.7 \%$ were presented at the overall less likely time point.

Every participant ran four experimental blocks of 160 trials each. Within two of the blocks, participants expected the targets at the first interval and vice versa in the other two blocks. The order was counterbalanced between the participants. One experimental session lasted approximately 1.5 hours in total. During the experiment, reaction times and response accuracy were recorded. Trials in which the participants failed to provide a response or in which the foot 
pedals were not correctly pressed were automatically discarded and repeated at the end of the block.

Before the beginning of the experiment, participants performed a training block of 48 trials, to familiarize with the experiment parameters and response mapping. The training had the same trial distribution as the first two experimental blocks. To facilitate the task learning, a feedback signal on error and correct responses was provided. Feedback was absent in the actual experiment. The data from the training were not analysed.

\section{Analysis}

Incorrect responses and RTs two standard deviations away from the individual mean were discarded from the analyses ( $<5 \%$ of all trials were excluded). In addition to RTs and accuracy, inverse efficiency scores ( $\mathrm{IE}=\mathrm{RT} /$ Proportion of correct responses) were calculated for each participant and condition. According to Bruyer and Brysbaert (2011), the use of IE scores makes sense especially if the error rate is not higher than $10 \%$, which is the case in our experiment, as revealed in the accuracy results. IE scores are interpreted like RTs and error rates, that is, the lower the score the more efficient is the processing of the event.

A repeated measures ANOVA was performed for RTs, accuracy and IEs with modality prevalence (primary, secondary), onset time $(1 \mathrm{~s}, 2.5 \mathrm{~s})$ and expected time point of the primary modality (early, late) as within participants factors, and the primary modality (vision, touch) as between participants factor. Statistics were performed with STATISTICA 8.0 (StatSoft Inc.; Tulsa, OK, USA). Student's t-tests were calculated as post-hoc analysis of the ANOVA. 


\section{Results}

\section{Reaction times}

We found a significant main effect of modality prevalence, with participants responding faster towards the primary, than the secondary modality (Fig. $2 \mathrm{~A} ; \mathrm{F}_{(1,27)}=58.56, \mathrm{p}<0.01, \eta \rho^{2}=0.68$ ). The main effect of temporal attention (time expectation) was also significant (Fig. 2B; $\left.\mathrm{F}_{(1,27)}=5.20, \mathrm{p}=0.03, \eta \rho^{2}=0.16\right)$, with overall faster responses at the expected time point. Importantly, we found a significant interaction between modality prevalence and time expectation (Fig. $2 \mathrm{C} ; \mathrm{F}_{(1,27)}=17,85, \mathrm{p}<0.01, \eta \rho^{2}=0.39$ ). While participants reacted significantly faster to primary targets presented at the expected, and overall more likely, time point compared to the unexpected time point $\left(\mathrm{t}_{28}=-3.75, \mathrm{p}<0.01\right)$, we found the reverse, nearly significant, pattern for targets in the secondary modality (slower RTs at expected vs. unexpected time point; $\left.\mathrm{t}_{28}=1.77, \mathrm{p}=0.09\right)$. This reveals a breach in cross-modal synergy and suggests, instead, a decoupling of time expectation across modalities. This decoupling was qualified by the significant triple interaction between interval, modality prevalence and expected time point $\left(F_{(1,27)}=7.32, p=0.01, \eta \rho^{2}=0.21\right)$, suggesting different patterns for the early and late time points (see Fig. 2D \& 2E). In order to follow up on this interaction, we ran separate ANOVAs for each (early and late) interval. Both time intervals revealed an interaction between modality prevalence and temporal expectation, just like in the main (pooled) data analysis. For the primary modality targets, time expectancy effects (faster RTs when the time point was the expected vs. unexpected one) were significant at the early time point $(1 \mathrm{~s})\left(\mathrm{t}_{28}=-2.51, \mathrm{p}=0.02\right)$ as well as for the second $(2.5 \mathrm{~s})$ time point $\left(\mathrm{t}_{28}=-2.42, \mathrm{p}=0.02\right)$. In the case of the secondary modality, however, such tendency levelled off $\left(\mathrm{t}_{28}=-0.79, \mathrm{p}=0.43\right)$ in the early time point and completely reversed in the second time point. That is, responses to targets in the secondary modality were significantly 
slower if participants expected a target in the primary modality in that interval, compared to the unexpected interval $\left(\mathrm{t}_{28}=2.71, \mathrm{p}=0.01\right)$. In summary, upon targets appearing after $1 \mathrm{~s}$, the secondary modality did not show the expectation effects of the primary modality. Furthermore, upon targets appearing after $2.5 \mathrm{~s}$, we found expectancy effects to abide to the relative likelihood of the secondary modality and counter to the likelihoods of the primary modality. This pattern was equivalent for both combinations of primary/secondary modalities (vision/touch, or touch/vision), since the interaction between primary modality, modality prevalence, expected time point and onset time did not reach statistical significance $\left(\mathrm{t}_{28}=1.95, \mathrm{p}=0.17, \eta \rho^{2}=0.07\right)$. However, for the sake of confirmation, we decided to run statistics on each modality combination separately. When touch was the primary modality, participants responded significantly faster to tactile targets if they were presented at the expected vs. unexpected time point $\left(\mathrm{t}_{13}=-4.26, \mathrm{p}<0.01\right)$. When touch was the secondary modality, no reaction time differences between expected and unexpected time points could be observed $\left(\mathrm{t}_{13}=1.261, \mathrm{p}=0.23\right)$. For vision, participants responded again significantly faster to visual primary targets at the expected time point $\left(\mathrm{t}_{14}=-3,12, \mathrm{p}<0.01\right)$, while for secondary visual targets no reaction time differences were found $\left(\mathrm{t}_{14}=1.36, \mathrm{p}=0.19\right)$.

In the overall ANOVA, we found a significant triple interaction between expected time point, modality prevalence and the primary modality $\left(\mathrm{F}_{(1,27)}=4.29, \mathrm{p}=0.048, \eta \rho^{2}=0.14\right)$, which was probably caused through the larger reaction time benefits for primary (vs. secondary) tactile targets than for primary visual. Finally, we found a significant interaction between primary modality and modality prevalence $\left(\mathrm{F}_{(1,27)}=10.97, \mathrm{p}<0.01, \eta \rho^{2}=0.29\right)$. Upon closer inspection of this pattern, the analysis did not reveal significant or marginal differences between the reaction times to vision and touch when they were the primary modality $\left(\mathrm{t}_{14}=1.26, \mathrm{p}=0.23\right)$, nor when 
they appeared as the secondary modality $\left(\mathrm{t}_{14}=-1.18, \mathrm{p}=0.26\right)$. Thus, the interaction between these variables within the ANOVA must be caused by non-significant trends in opposing directions. Because the underlying cause of this interaction is orthogonal to the interests of this study, it will not be discussed any further.

\section{Accuracy}

Overall, response accuracy was very high (on average 95.3\% $\pm 5.2 \%$ ), meaning that participants were able to successfully perform the task and distinguish between single and double pulse stimuli, as instructed. We found a significant main effect of modality prevalence $\left(\mathrm{F}_{(1,27)}=5,41\right.$, $\mathrm{p}=0.03, \eta \rho^{2}=0.17$, with slightly more accurate responses towards primary than secondary targets. The main effect of onset time was significant as well $\left(\mathrm{F}_{(1,27)}=6,94, \mathrm{p}=0.01, \eta \rho^{2}=0.21\right)$. Participants responded more accurately to targets presented at the late time interval, compared with responses to early targets. Additionally, we found a significant interaction between primary modality, modality prevalence and onset time $\left(F_{(1,27)}=5,72, p=0.02, \eta \rho^{2}=0.18\right)$, that is when the primary modality was touch, there was a trend toward more accurate responses in the primary modality for early onset times $\left(\mathrm{t}_{13}=2.09, \mathrm{p}=0.06\right)$ as well as a similar trend towards more accurate responses in the primary modality for the late interval $\left(\mathrm{t}_{13}=1.93, \mathrm{p}=0.08\right)$. In contrast, if vision was the primary modality, no significant accuracy differences between the primary and secondary modality were found for both early $\left(\mathrm{t}_{14}=1.48, \mathrm{p}=0.16\right)$ and late onset times $\left(\mathrm{t}_{14}>-0.01\right.$, $\mathrm{p}=0.99$ ). Despite this pattern of effects is consistent with the one seen for the RTs, due to the overall high percentages (leading to reduced variability) and the very small differences, accuracy effects will not be interpreted any further. We refer the reader to the inverse efficiency scores, 
reported below, which incorporate accuracy and RTs in one measure. No other main effect or interaction reached significance.

\section{Inverse Efficiency}

For Inverse Efficiency (IE) scores, the main effect of modality prevalence was highly significant $\left(F_{(1,27)}=47.36, p<0.01, \eta \rho^{2}=0.64\right)$, with participants responding more efficiently to the primary than to the secondary modality (Fig. 3A). This modality prevalence effect was present in both, vision $\left(\mathrm{t}_{14}=-2.59, \mathrm{p}=0.02\right)$ and touch $\left(\mathrm{t}_{13}=-7.45, \mathrm{p}<0.01\right)$ individually, but stronger if touch was the primary modality as revealed by the interaction between modality prevalence and primary modality $\left(\mathrm{F}_{(1,27)}=9,21, \mathrm{p}<0.01, \eta \rho^{2}=0.25\right)$. The main effect of onset time was significant as well $\left(\mathrm{F}_{(1,27)}=4,79, \mathrm{p}=0.037, \eta \rho^{2}=0.15\right)$, reflecting that responses to targets appearing at the late time point were more efficient than responses to targets presented at the early time point .

Like in the RT analysis, for IE scores the critical interaction between modality prevalence and expected time was significant $\left(\mathrm{F}_{(1,27)}=16,27, \mathrm{p}<0.01, \eta \rho^{2}=0.38\right)$, attesting to the decoupling between temporal expectations across modalities (Fig. 3B). For the primary modality, participants responded more efficiently if the target appeared at the expected time point $\left(\mathrm{t}_{28}=-3.948, \mathrm{p}<0.01\right)$, while for the secondary modality, a nearly significant trend in the opposite direction, that is more efficient responses at the unexpected onset times, was observed $\left(\mathrm{t}_{28}=1.87\right.$, $\mathrm{p}=0.07)$.

Investigating this pattern for each modality separately, we found that for touch as primary modality, participants responded more efficiently if the stimulus was presented at the expected (vs. unexpected) time point $\left(\mathrm{t}_{13}=-3.125, \mathrm{p}<0.01\right)$. For secondary tactile targets a marginal trend towards more efficient responses at the unexpected time point was observable $\left(\mathrm{t}_{13}=1.833\right.$, 
$\mathrm{p}=0.090$ ). For vision, participants responded more efficiently towards primary visual targets at expected vs. unexpected time points $\left(\mathrm{t}_{14}=-3.17, \mathrm{p}<0.01\right)$, whilst no response differences between expected an unexpected onset times for visual secondary targets were observed $\left(\mathrm{t}_{14}=0.71, \mathrm{p}=0.49\right)$. Neither the 3-way interaction between modality prevalence, expected time and onset, nor any other effect or interaction further reached significance.

In conclusion, our IE results pattern in the same direction as the reaction time results, confirming that there was no trade-off between accuracy and reaction times in participants' performance. For the upcoming discussion, we will therefore mainly focus on the reaction time results.

\section{Discussion}

According to the present results, temporal attention is not subject to a strong cross-modal synergy and, instead, it can be deployed in a relatively independent manner for separate sensory modalities. Considering only the primary modality, our results are conform to the well-known effect of attention to time in single modality studies (Coull and Nobre, 1998; Miniussi et al., 1999; Griffin et al., 2001; Correa et al., 2004), meaning that performance improves for targets at expected, compared to unexpected time points. ${ }^{1}$

\footnotetext{
${ }^{1}$ It is noteworthy that we did not find a clear benefit in terms of accuracy, as Correa \& Nobre (2008) did. However this difference can be explained due to our overall very high accuracy.
} 
The important aspect of the present results is that the secondary, overall less likely modality did not follow the orienting of attention induced by the primary modality. Instead, in trials where a target was expected in the early time interval but not presented, modality expectation quickly reoriented towards the secondary modality at the upcoming late interval. In trials where a target appeared unexpectedly early, responses to the primary modality suffered a decrease in performance and yet, no particular performance differences arose between temporally expected vs. unexpected targets in the secondary modality.

It is noteworthy that the conclusions supported by the present data seem to be at variance with the findings of Lange \& Röder (2006), who reported that the secondary modality was modulated in the same direction as the primary modality. Instead, what our results suggest is that the deployment of temporal attention is not coupled across modalities. Our finding also stands in contrast with the more often studied case of spatial attention (Spence \& Driver, 1996; Eimer, 1999), according to which orienting towards one particular modality and location in space leads to benefits (i.e., faster reaction times) for stimuli of other modalities at that location, even when events in this other modality are in fact more likely to appear at a different spatial location. That is, for spatial attention humans do seem to allocate resources towards the most likely location for all possible modalities, at the expense of poorer modality selectivity (i.e., even when orienting to other, infrequent, modalities is disadvantageous for overall efficiency; Eimer, 1999; Macaluso, 2010). In contrast, according to the present data, in the case of time participants can selectively deploy their attention to particular instants and modalities. For example, we did not find a benefit at the overall most likely time of stimulus appearance, but a benefit (significant or nearly significant, depending on condition and measured variable) at the relatively more likely time for that particular modality. 
Despite the direction of cross-modal temporal attention stands in contrast with the pattern of spatial attention effects, in terms of strength of orienting in the primary and secondary modality however, our results seem to be similar to previous spatial attention findings (e.g. Spence \& Driver, 1996). In particular, both in previous spatial attention findings and our results, the orienting effects across modality (i.e. in the secondary modality) manifest in a reduced manner compared to unimodal attention effects or effects in the primary modality.

Another point of interest in our results is that modality selectivity in temporal attention depended on whether the expected time point of the primary modality was early or late (a pattern that was most clearly seen in RTs). This is relevant because many of the previous studies could only analyse data from the early time point due to the confound often present in temporal attention studies due to the changing hazard rate throughout the trial (Coull \& Nobre, 1998; Miniussi et al., 1999; Griffin et al., 2002; Lange \& Röder, 2006). Different from other studies, we did at least reduce this confound by making the occurrence of events at the last time point not completely predictable. In this respect, an interesting and novel observation in our RT data is that the tendency for separable expectations across modalities was stronger at the late interval. Note that this pattern rules out that our effect merely show a reorientation of attention within the time scale. In other words, that attention would be always focused on the overall most likely time point, independent of the modality. According to this strategy, if the most likely time point of stimulus occurrence passed without a target, participants would simply focus attention on the next likely time point. However, the 3-way interaction found between modality prevalence, expected time point and onset time reveals that the selective effects in reaction time was strongest for the late (2.5s) stimulus onset, while no difference between expected and unexpected 
event occurrence was observed for early (1s) onset times. When the early onset was overall less likely, then we found neither performance increases, nor decreases for the secondary modality.

We argue that, based on the present pattern of results, endogenous attention to time and to modality may unfold at a slightly different time courses. In particular, when attention must be deployed immediately (i.e., first time interval after the cue) modality selectivity is poorer. That is, resources are allocated in a less specific (perhaps less efficient) way so that the possible expectation effects on the primary modality will impose some automatic orienting to the secondary, unlikely, modality. When attention must be deployed at later time points, then modality selectivity is more efficient, and fully sensitive to relative probability differences across modalities. Thus, more specifically, we might first deploy our temporal expectation, which leads to more general reaction time benefits, before we deploy our modality expectation.

One might argue here that secondary modality targets were just easy or that temporal attention was not manipulated effectively. However, primary modality significant expectation results make us rule out this alternative. Indeed, when temporal attention was deployed at the long interval, then both, expectation in time as well as expectation to modality, are more solidly deployed, so that the sensitivity to more subtle probability modulations on the less likely secondary modality played an effective modulation. Note that relative differences in difficulty across modalities cannot easily explain this pattern, since both visual and tactile targets played the role of secondary modality across these data.

This hypothesis does not only account for our present pattern of results, but also helps explain the above mentioned differences between our present findings and the study by Lange and Röder (2006), who concluded that there was a benefit of attending time for both, the attended and the unattended modality. In particular, because of the discussed artefact introduced by the increasing 
hazard rate throughout the trial, Lange and Röder did not analyse the late time intervals, whereas in our experiment, the decoupling between modalities in time was more evident, precisely, at later intervals. According to the possible time course of temporal expectation and attention to modality, discussed above, one could think that Lange and Röder might have limited their focus of enquiry to an initial stage of the process whereby an early attention shift select for time but not modality. This fits well with the fact that Lange and Röder used shorter intervals $(600 \mathrm{~ms}$ or $1200 \mathrm{~ms}$ ) after trial onset, whereas we used longer ones, which might have given the participant even more time to fully orient their attention to time as well as modality. This would explain why, the secondary modality followed a synergistic pattern in the first interval for Lange and Röder $(600 \mathrm{~ms})$ and started to level off in our first interval $(1000 \mathrm{~ms})$ with no particular advantage or disadvantage. It would also explain the more evident modality selectivity found in our study in the second interval $(2500 \mathrm{~ms})$.

There are some other differences between the experiment of Lange and Röder and our experiment, which may underlie their disparate outcomes, though it is less clear how. For example, Lange and Röder used auditory and tactile stimuli, while we used visual and tactile stimuli. It is therefore a possibility that different attention links between different pairs of modalities abide to different rules (see Driver \& Spence, 1998b; Spence \& McDonald, 2004), for an example relating to cross-modal exogenous attention. In addition, Lange and Röder used a tactile warning to signal the start of each trial, a modality which was also used as one of their target modalities in the task. This may have influenced the resulting tuning of attention to a modality, so that when the visual modality was primary, participants still had to attend to touch to be aware of trial initiation and then quickly switch to vision. For this reason, we used an 
auditory tone as trial onset warning, which was an orthogonal marker to minimize modality biases.

A relevant outcome of the present study is that it points to a basic feature of temporal attention which would reveal a fundamental distinction between attention to time and attention to space. Whilst, according to many previous demonstrations, spatial attention tends to affect attended and unattended sensory modalities in a synergistic manner, this is not necessarily the case for temporal attention. Instead, selection in time seems to tune benefits of attended stimuli at their most likely temporal onset. These differences in behavioural expression are non-conflicting with known differences in neural correlates of spatial and temporal attention. For example, ERP studies show that while spatial attention typically leads to clearly lateralized amplitude enhancements of the P1 and N1 components of the attended stimuli (e.g. Eimer \& Schröger, 1998; Teder-Sälejärvi et al., 1999; Eimer \& Driver, 2000, McDonald et al., 2005; Störmer et al., 2009), temporal attention reflects in a spatially widespread, less lateralized activation pattern on the scalp (Griffin et al., 2002). In terms of ERP components, both temporal and spatial attention have been shown to affect the P1 (Correa et al., 2006) and the N1 component (Miniussi et al., 1999; Griffin et al., 2002;Lange \& Röder, 2006; Sanders \& Astheimer, 2008; Astheimer \& Sanders, 2009; Lampar \& Lange, 2011; Lange, 2012), but only temporal attention has been shown to modulate the N2 (Griffin et al., 2002; Sanders \& Astheimer, 2008) and the P3 components (Miniussi et al., 1999; Griffin et al., 2002; Lampar \& Lange , 2011). At the level of oscillatory dynamics, it is well known that orienting spatial and temporal attention both lead to amplitude modulations of EEG activity at low frequency bands in the pre-stimulus period (Händel et al., 2011; Hanslmayr et al., 2011; Foxe \& Snyder, 2011). For 
instance, the deployment of visual spatial attention leads to contralateral power decreases and ipsilateral power increases in the alpha band over the occipital cortex (e.g. Worden et al., 2000; Sauseng et al., 2005; Thut et al., 2006; Trenner et al., 2008; Gould et al., 2011; Händel et al, 2011; Capilla et al., 2012). In cross-modal studies, the low-frequency spatial modulation, due to orienting attention to the expected location and away from the unexpected one, does also occur between the corresponding sensory cortices of the expected vs. unexpected modality (Bauer et al., 2012). However, it is less clear how this inter-hemispheric modulation could encompass a function of temporal selective attention. Instead, because brain oscillations represent events in time itself (Buzsáki, 2006), one could assume that temporal attention might modulate the readiness towards an attended modality by modulating the ongoing oscillatory patterns within sensory-specialised brain regions (van Ede, 2011). More evidence for distinct underlying neural mechanisms comes from more spatially precise imaging models. Studies, conducted with fMRI (Brunetti et al., 2008; Silk et al., 2010; Li et al., 2012; Yang \& Mayer, 2013) and PET (Corbetta et al., 1993; Nobre et al., 1997) show that spatial and temporal attention orienting engages some common brain areas (like the prefrontal cortex, the insular cortex, the dorsolateral premotor cortex or the inferior parietal lobe), though there are some important differences also. Indeed, according to extant evidence, spatial but not temporal attention tasks often lead to activation of the frontal eye fields, ventrolateral premotor cortex the intraparietal sulcus, the anterior cingulate cortex and subcortical regions. Temporal attention tasks, instead, have been more often shown to lead to activation in the middle temporal gyrus, the superior occipital gyrus and the cerebellum (Coull \& Nobre, 1998; Davranche et al., 2011; Li et al., 2012). 
The findings discussed above, obtained with various methods indicate similarities but also profound differences in the neural mechanisms underlying temporal and spatial attention. This must in part be due to the dramatic differences between encoding the dimensions of space and time. Temporal attention usually involves processing of time-shifted events, while spatial attention involves competition between (possible) events occurring at about the same time. In other words, during spatial attention a participant usually has to focus attention on one out of several isochronous potential events, which are all competing for processing resources at the same time (Desimone and Duncan, 1995). In contrast, while focusing attention in time, potentially relevant events are anisochronous. Depending on the temporal difference between two events, temporal attention can allocate resources flexibly and dynamically to adapt efficiently towards task demands. Under the light of this framework, it seems only logical that temporal and spatial attention may share some similarities but also display very different outcomes at the behavioural level. While in spatial attention, the isochrony of possible events tends to create cross-modal linkage to optimize resources, in temporal attention events can be cross-modally decoupled since they are anisochronous and resources can be allocated dynamically.

Within the present study, we manipulated the participants attention through different target probabilities, in terms of its onset times and modality. For example, a more likely modality is also more relevant for participants and therefore it will necessarily drive their endogenous attention. On the other hand different target probabilities lead also to different target predictabilities and therefore modulate the participants' expectation (Lange, 2013). Thus, like in most other temporal attention studies, we are well aware that for the moment these findings must be attributed to a combination of attention and expectation effects. Although attention and 
expectation can be functionally distinguishable and lead to different effects (Summerfield \& Egner, 2009), it is not the goal of this study to measure their different contributions.

This study addressed whether orienting attention in time leads to synergistic behavioural cross-modal effects, as shown previously for spatial attention (i.e., Spence \& Driver, 1996) and more recently suggested for temporal attention (Lange \& Röder, 2006). We found that processing of a likely (primary) modality is enhanced at its expected (most likely overall) time point. This is an expected result. Instead, for targets in the less likely (secondary) modality we found that allocation of temporal attention was sensitive to relative, local occurrence probabilities, even when these were in opposition with the occurrence probabilities of an overall most likely event modality. This outcome is in stark contrast to results obtained by previous studies of spatial attention, where both, primary and secondary targets, are enhanced at the expected side of the primary modality (Spence \& Driver, 1996; Eimer, 1999). Our results can also not solely be explained by reorienting attention in time. In that case, one should have observed that for the early time point, the secondary and primary modality would both be modulated in the same direction through temporal attention, whilst for the late time point; the secondary modality should not follow the temporal modulation of the primary modality, but instead be faster if the late time point was not expected. Therefore, we conclude that our results seem to point towards a general different mechanism of spatial and temporal attention, which seem to be supported by the various findings obtained in studies using physiological recordings as ERPs and fMRI. 


\section{Acknowledgements}

This research was funded by the Spanish Ministry of Science and Innovation (PSI2010-15426), the Comissionat per a Universitats i Recerca del DIUE-Generalitat de Catalunya (SRG2009-092) and the European Research Council (StG-2010 263145) to S.S.F. 


\section{References}

Astheimer, L. B. \& Sanders, L. D. ( 2009). Listeners modulate temporally selective attention during natural speech processing. Biol. Psychol., 80, 23-34.

Bauer, M., Kennett, S. \& Driver, J. (2012). Attentional selection of location and modality in vision and touch modulates low-frequency activity in associated sensory cortices.

J. Neurophysiol., 107(9), 2342-51.

Brunetti, M., Della, P. S., Ferretti, A., Del, G. C., Cianflone, F., Belardinelli, P., Caulo, M., Pizzella, V., Olivetti, B. M. \& Romani, G. L. (2008). A frontoparietal network for spatial attention reorienting in the auditory domain: a human fMRI/MEG study of functional and temporal dynamics. Cereb. Cortex, 18, 1139-1147.

Bruyer, R. \& Brysbaert, M. (2011). Combining Speed and Accuracy in Cognitive Psychology: Is the Inverse Efficiency Score (IES) a better Dependent Variable than the Mean Reaction Time (RT) and the Percentage of Errors (PE)? Psychol. Belg., 51, 5-13.

Buzsáki, G. (2006). Rhythms of the Brain. Oxford University Press.

Capilla, A., Schoffelen, J.-M., Paterson, G., Thut, G. \& Gross, J. (2012). Dissociated $\alpha$-Band Modulations in the Dorsal and Ventral Visual Pathways in Visuospatial Attention and Perception. Cereb. Cortex, 10, 1-12.

Carrasco, M. (2012). Visual attention : The past 25 years. Vision Res., 51(13), 1484-1525.

Corbetta, M., Miezin, F. M., Shulman, G. \& Petersen, S. E. (1993). A PET study of visuospatial attention. J. Neurosci., 13, 1202-1226. 
Corbetta, M. \& Shulman, G. L. (2002). Control of goal-directed and stimulus-driven attention in the brain. Nature Rev. Neurosci., 3(3), 201-15.

Correa, A., Lupiánez, J., Milliken, B. \& Tudela, P. (2004). Endogenous temporal orienting of attention in detection and discrimination tasks. Percept. Psychophys., 66, 264-278.

Correa, A., Lupiánez, J., Madrid, E. \& Tudela, P. (2006). Temporal attention enhances early visual processing: a review and new evidence from event-related potentials. Brain Res., 1076, $116-128$.

Coull, J. T. \& Nobre, A. C. (1998). Where and when to pay attention: the neural systems for directing attention to spatial locations and to time intervals as revealed by both PET and fMRI. $J$. Neurosci., 18(18), 7426-35.

Coull, J. T. \& Nobre, A. C. (2008). Dissociating explicit timing from temporal expectation with fMRI. Current Opinion in Neurobiology, 18(2), 137-44.

Davranche, K., Nazarian, B., Vidal, F. \& Coull, J. (2011). Orienting attention in time activates left intraparietal sulcus for both perceptual and motor task goals. J. Cognitive Neurosci., 23(11), $3318-30$.

Desimone, R. \& Duncan, J. (1995) Neural mechanisms of selective visual attention. Ann. Rev. Neurosci., 18, 193-222.

Driver, J. \& Spence, C. (1998a).Crossmodal attention. Curr. Opin. Neurobiol., 8, 245-253.

Driver, J. \& Spence, C. (1998b). Attention and the crossmodal construction of space.

Trends Cogn. Sci., 2(7), 254-62. 
Eimer, M. (1999) Can attention be directed to opposite locations in different modalities? An ERP study. Clin. Neurophysiol., 111, 1252-1259.

Eimer, M. \& Driver, J. (2000). An event-related brain potential study of cross-modal links in spatial attention between vision and touch. Psychophysiology, 37, 697-705.

Eimer, M. \& Schröger, E. (1998). ERP effect of intermodal attention and cross-modal links in spatial attention. Psychophysiology, 35, 313-327.

Eimer, M., van Velzen, J. and Driver, J. (2002).Cross-modal interactions between audition, touch and vision in endogenous spatial attention: ERP evidence on preparatory states and sensory modulations. J. Cognitive Neurosci., 14, 254-271.

Foxe, J. J. \& Snyder, A. C. (2011). The Role of Alpha-Band Brain Oscillations as a Sensory Suppression Mechanism during Selective Attention. Front. Psychol., 2, 154.

Gould, I. C., Rushworth, M. F. Nobre, A. C. (2011). Indexing the graded allocation of visuospatial attention using anticipatory alpha oscillations. J. Neurophysiol., 105, 1318-1326. Griffin, I. C., Miniussi, C. \& Nobre, A. C. (2001). Orienting attention in time. Front. Biosci., 6, $660-671$.

Griffin, I. C., Miniussi, C. \& Nobre, A. C. (2002). Multiple mechanisms of selective attention: Differential modulation of stimulus processing by attention to space or time. Neuropsychologia, 40, 2325-2340.

Hanslmayr, S., Gross, J., Klimesch, W. \& Shapiro, K. L. (2011). The role of $\alpha$ oscillations in temporal attention. Brain Res. Rev., 67, 1-2, 331-43. 
Händel, B. F., Haarmeier, T. \& Jensen, O. (2011). Alpha Oscillations Correlate with the Successful Inhibition of Unattended Stimuli. J. Cognitive Neurosci., 23(9), 2494-502.

Jones M. R. (2004). Attention and timing. In Ecological Psychoacoustics.Edited by Neuhoff J. Elsevier Academic Press.

Jonides, J. (1981). Voluntary versus automatic control over the mind's eye's movement. In: Long, J.B., Baddely, A.D. (Eds.), Attention and Performance, vol. IX. Lawrence Erlbaum, Hillsdale, NJ, 187-203.

Kennett, S., Eimer, M., Spence, C. \& Driver, J. (2001).Tactile-visual links in exogenous spatial attention under different postures: Convergent evidence from psychophysics and ERPs. $J$. Cognitive Neurosci., 13, 462-478.

Krumbholz, K., Nobis, E. A., Weatheritt, R. J. \& Fink, G. R. (2009). Executive control of spatial attention shifts in the auditory compared to the visual modality. Hum. Brain Mapp., 30, 14571469.

Lampar, A. L. \& Lange, K. (2011). Effects of temporal trial-by-trial cuing on early and late stages of auditory processing: evidence from event-related potentials.

Atten. Percept. Psychophys., 73, 1916-1933.

Lange, K. (2012). The N1 effect of temporal attention is independent of sound location and intensity: implications for possible mechanisms of temporal attention. Psychophysiology, 49(11), $1468-1480$.

Lange, K. (2013). The ups and downs of temporal orienting: a review of auditory temporal orienting studies and a model associating the heterogeneous findings on the auditory N1 with opposite effects of attention and prediction. Front. Hum. Neurosci., 7, 263. 
Lange, K. \& Röder, B. (2006). Orienting attention to points in time improves stimulus processing both within and across modalities. J. Cognitive Neurosci., 18(5), 715-29.

Li, C., Chen, K., Han, H., Chui, D. \& Wu, J. (2012). An FMRI study of the neural systems involved in visually cued auditory top-down spatial and temporal attention. PloS one, 7(11), e49948.

Luck, S. J., Hillyard, S. A., Mouloua, M., Woldorff, M. G., Clark, V. P. \& Hawkins, H. L. (1994). Effects of spatial cueing on luminance detectability: Psychophysical and electrophysiological evidence for early selection. J. Exp. Psychol. Human, 20(4), 887-904.

Macaluso, E., Frith, C. D. \& Driver, J. (2000). Modulation of human visual cortex by crossmodal spatial attention. Science, 289, 1206-1208.

Macaluso, E., Frith, C. D. \& Driver, J. (2002). Directing attention to locations and to sensory modalities: multiple levels of selective processing revealed with PET. Cereb. Cortex, 12, 357368.

Macaluso,E. (2010) Orienting of spatial attention and the interplay between the senses. Cortex, 46(3), 282-297.

McDonald, J. J., Teder-Sälejärvi, W. A. \& Hillyard, S. A. (2000).Involuntary orienting to sound improves visual perception. Nature, 407, 906-908.

McDonald, J. J., Teder-Sälejärvi, W. A., Di Russo, F. \& Hillyard, S. A. (2005). Neural basis of auditory-induced shifts in visual time-order perception. Nat. Neurosci., 8, 1197-1202.

Meredith, M. A. (2002). On the neuronal basis for multisensory convergence: A brief overview. Cognitive Brain Res., 14, 31-40. 
Miniussi, C., Wilding, E. L., Coull, J. T. \& Nobre, A. C. (1999). Orienting attention in time. Modulation of brain potentials. Brain, 122, 1507-1518..

Nobre, A. C., Correa, A. \& Coull, J. T. (2007). The hazards of time. Curr. Opin. Neurobiol., 17(4), 465-470.

Nobre, A. C., Gitelman, D. R., Sebestyen, G. N., Meyer, J., Frackowiak, R. S. J., Frith, C. D. \& Mesulam, M. M. (1997). Functional localisation of the system for visuospatial attention using positron emission tomography. Brain, 120, 515-533.

Posner, M. I., Snyder, C. R. \& Davidson, B. J. (1980). Attention and the detection of signals. J. Exp. Psychol., 109(2), 160-74.

Rohenkohl, G., Cravo, A. M., Wyart, V. \& Nobre, A. C. (2012). Temporal Expectation Improves the Quality of Sensory Information. J. Neurosci., 32(24), 8424-8428.

Sanders, A. F.(1975). The foreperiod effect revisited. Q. J. Exp. Psychol., 27, 591-598.

Sanders, L. D. \& Astheimer, L. B. (2008). Temporally selective att0ention modulates early perceptual processing: event-related potential evidence. Percept. Psychophys., 70, 732-742.

Santangelo, V., Olivetti Belardinelli, M., Spence, C. \& Macaluso, E. (2009). Interactions between voluntary and stimulus-driven spatial attention mechanisms across sensory modalities. $J$. Cognitive Neurosci., 21(12), 2384-2397 
Sauseng, P., Klimesch, W., Stadler, W., Schabus, M., Doppelmayr, M., Hanslmayr, S., Gruber, W. R. \& Birbaumer, N. (2005). A shift of visual spatial attention is selectively associated with human EEG alpha activity. Eur. J. Neurosci., 22, 2917-2926.

Silk, T. J., Bellgrove, M. A, Wrafter, P., Mattingley, J. B. \& Cunnington, R. (2010). Spatial working memory and spatial attention rely on common neural processes in the intraparietal sulcus. NeuroImage, 53(2), 718-24.

Spence, C. \& Driver, J. (1996). Audiovisual links in endogenous covert spatial attention. J. Exp. Psychol. Human, 22(4), 1005-1030.

Spence, C. \& Driver, J. (1997). Audiovisual links in exogenous covert spatial orienting. Percept. Psychophys., 59, 1-22.

Spence, C. \& McDonald., J. J. (2004). The crossmodal consequences of the exogenous spatial orienting of attention. In The Handbook of Multisensory Processing. Calvert, G., A., Spence, C., \& Stein, B., E., 3-25. MIT Press. Cambridge, MA.

Spence, C., Nicholls, M.E., Gillespie, N. \& Driver, J. (1998).Cross-modal links in exogenous covert spatial orienting between touch, audition and vision. Percept. Psychophys., 60, 544-557.

Spence, C., Nicholls, M. E. \& Driver, J. (2001). The cost of expecting events in the wrong sensory modality. Percept. Psychophys., 63(2), 330-336.

Spence C, Pavani F \& Driver J. (2000). Crossmodal links between vision and touch in covert endogenous spatial attention. J. Exp. Psychol. Human, 26, 1298-1319. 
Störmer, V. S., McDonald, J. J. \& Hillyard, S. A. (2009). Cross-modal cueing of attention alters appearance and early cortical processing of visual stimuli. PNAS, 106, 22456-22461 Summerfield, C. \& Egner, T. (2009). Expectation (and attention) in visual cognition. Trends Cogn. Sci. 13, 403-409.

Teder-Sälejärvi, W. A., Münte, T. F., Sperlich, F.-J. \& Hillyard, S. A. (1999). Intra-modal and cross-modal spatial attention to auditory and visual stimuli: An event-related brain potential (ERP) study. Cognitive Brain Res., 8, 327-343.

Thut, G., Veniero, D., Romei, V., Miniussi, C., Schyns, P. \& Gross, J. (2011). Rhythmic TMS causes local entrainment of natural oscillatory signatures. Current Biol., 21, 1176-1185. Trenner, M. U., Heekeren, H. R., Bauer, M., Rossner, K., Wenzel, R., Villringer, A. \& Fahle, M. (2008). What happens in between? Human oscillatory brain activity related to crossmodal spatial cueing. PLoS One, 3:e1467.

Treue, S. (2003). Visual attention: the where, what, how and why of saliency. Curr. Opin. Neurobiol., 13(4), 428-432.

van Ede, F., De Lange, F. P., Jensen, O. \& Maris, E. (2011). Orienting Attention to an Upcoming Tactile Event Involves a Spatially and Temporally Specific Modulation of Sensorimotor Alphaand Beta-Band Oscillations. J. Neurosci., 31(6), 2016-2024.

Westheimer, G. \& Ley, E. (1996). Temporal uncertainty effects on orientation discrimination and stereoscopic thresholds. J. Opt. Soc. Am., 13, 884-886.

Worden, M. S., Foxe, J. J., Wang, N. \& Simpson, G.V. (2000). Anticipatory biasing of visuospatial attention indexed by retinotopically specific alphaband electroencephalography increases over occipital cortex. J. Neurosci., 20, 1-6. 
Yang, Z. \& Mayer, A. R. (2013). An event-related FMRI study of exogenous orienting across vision and audition. Hum. Brain Mapp., 34, 1097-0193.

Yeshurun, Y., \& Carrasco, M. (1998). Attention improves or impairs visual performance by enhancing spatial resolution. Nature, 396(6706), 72-75.

Fig. 1: Schematic description of the task. A) Depiction of the experimental setup. Participants rested their hands underneath a cardboard box, which contained two adjustable stimulation LEDs that were used for target presentation (1) and fixation (2). The stimulation tapper (3) was strapped to the index finger of the participants. Two foot pedals underneath one of the participants' foot were used for the response delivery. B) Schematic timeline of the trial. Every trial started with an auditory warning tone $(100 \mathrm{~ms}$ duration), followed by either visual or a tactile stimulus after $1000 \mathrm{~ms}$ or $2500 \mathrm{~ms}$. The direction of attention in time and modality was manipulated by manipulating the likelihood of stimulus appearance. Most targets would occur within one of the two modalities (fixed between participants) and at one of the two onset times (fixed between blocks). Furthermore, targets in the other, less frequent modality, would be relatively more likely at the overall less likely time point. Within some trials, no target was presented to prevent to build up of temporal certainty. C) Trial type distribution within a given experimental block. Every block consisted of 160 trials, of which $55 \%$ (88 trials) would contain a target at the expected time point. At the expected time point, approximately $86 \%$ (76 trials) would be presented in the primary modality and 13\% (12 trials) in the secondary modality. Approximately $22 \%$ of trials (36 trials) are presented at the unexpected time point. At this point, only $33 \%$ (12 trials) of trials contained a primary 
modality target and $66 \%$ (24 trials) a target in the secondary modality. The remaining $22 \%$ of the total number of trials ( 36 trials) contained no target at all.

Fig. 2: Inter-participant mean Reaction Times (RTs). All error bars account for the standard error of the mean. A) Overall mean RTs for primary and secondary targets. Participants responded significantly faster to targets of the primary modality $(0.680 \mathrm{~s})$, than to the secondary modality (0.784s). B) Overall mean RTs for targets presented in the expected and unexpected time points. Responses where significantly faster if the target onset time was expected (0.713s), compared when it was not expected $(0.731 \mathrm{~s})$. C) Interaction between attention to time and modality. Responses to targets in the primary modality (light grey) were significantly faster at expected, vs. unexpected time points $(0.655 \mathrm{~s}$ vs. $0.705 \mathrm{~s})$, whereas there was a trend for the secondary modality (dark grey) towards slower RTs at overall expected vs. unexpected time points ( $0.771 \mathrm{~s}$ vs. $0.756 \mathrm{~s}$, respectively). D) Average RTs towards stimuli presented at $1 \mathrm{~s}$ as a function of modality prevalence. RTs to primary modality targets (light grey) were significantly faster if participants expected a target to appear at that time point, than when they did not expect the target to appear at that moment $(0.656 \mathrm{~s}$ vs. $0.708 \mathrm{~s}$, respectively). For targets in the secondary modality (dark grey), there was no significant difference (0.757s vs. 0.769s, for overall expected vs. unexpected time points). E) Average RTs towards stimuli presented at $2.5 \mathrm{~s}$ as a function of modality prevalence. RTs to primary modality targets (light grey) were significantly faster if the participants expected a target to appear at that time point than when the time point of the most likely target appearance had already past $(0.657 \mathrm{~s}$ vs. 
0.733s). Interestingly for targets in the secondary modality (dark grey), participants RTs were significantly slower if the time was expected (i.e., primary modality targets most likely to appear) than when it was unexpected overall ( $0.784 \mathrm{~s}$ vs. $0.743 \mathrm{~s})$.

Fig. 3: Inverse Efficiency (error bars account for the standard error of the mean). A) Inverse efficiency scores for primary and secondary modality targets. Participants responded more efficiently towards primary than secondary modality targets $(0.708 \mathrm{~s}$ vs. $0.849 \mathrm{~s})$. B) Interaction between attention to time and modality. For targets in the primary modality (light grey), responses were significantly more efficient at the expected compared to the unexpected onset time $(0.680 \mathrm{~s}$ vs. $0.736 \mathrm{~s})$. Instead, targets in the secondary modality (dark grey) showed a trend towards more effective responses at the unexpected (at which the secondary modality was the most likely modality to appear), compared to expected time points ( $0.805 \mathrm{~s}$ vs. $0.832 \mathrm{~s})$. 